Brit. J. prev. soc. Med. (1975), 29, 18-21

\title{
A test for seasonality of events with a variable population at risk*
}

\author{
S. D. WALTER AND J. M. ELWOOD \\ Department of Epidemiology and Community Medicine, University of Ottawa, Canada
}

\begin{abstract}
Walter, S. D. and Elwood, J. M. (1975). British Journal of Preventive and Social Medicine, 29, 18-21. A test for seasonality of events with a variable population at risk. A statistical significance test to detect seasonality of epidemiological events is described. The method is similar to that of Edwards, but makes it possible to allow for an arbitrary pattern of variation in the population at risk, and also for the unequal lengths of time sectors of a cycle of seasons (e.g., months of a year). From the test it is possible to estimate the amplitude of seasonal variation and the time at which the maximum occurs in a postulated simple harmonic fluctuation; the adequacy of the description of the data by a curve of this kind may be evaluated using a goodness-of-fit test. A numerical example of the calculations is given using some anencephalus data, and the results are compared with those of alternative tests.
\end{abstract}

The description of incidence rates in terms of seasonal variation or cyclic trends is an important objective in many epidemiological problems, and the use of statistical tests to detect such variation is widespread; in particular, the test proposed by Edwards (1961) has occupied a central place in analyses of this kind, having been applied to a wide variety of epidemiological data, and also in other disciplines. The test has been criticized by Wehrung and Hay (1970) for being sensitive to occasional extreme values in the data, and a non-parametric alternative test has been suggested (Hewitt et al., 1971) when the sample size is small. A further limitation of Edwards' test is that it does not take account of the size of the population at risk, when it is usually seasonality of rates rather than pure numbers which is of interest to epidemiologists. Examples of problems where the variable population at risk is a consideration are provided by the analysis of congenital malformations with a seasonably variable number of total births, or the incidence of postoperative deaths for an elective surgical procedure demonstrating seasonal variation because of hospital administrative arrangements. Making allowances for this factor is important in a statistical evaluation of seasonality as may be seen by two extreme examples: first, supposing that a cyclic trend of disease events was in phase with the trend for the population at risk (i.e., the maxima occur at approximately the same timè), then a significant cyclic trend estimated solelyฏ from the numbers of events might have been $\bar{\circ}$

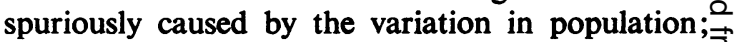
second, if the two trends were completely out ofo phase, a true cyclic variation in rates may be $e_{\supset}^{J}$ masked by the cancelling effect of population.

Although Edwards suggests the use of correction factors to adjust his test for variable populations at risk, it is proposed here to extend the method? somewhat more rigorously. A further generalization 3 will be in the use of time sectors (e.g., months of the year) which are not necessarily equal, as is required by Edwards' formulation. An illustrative example is given using some data on anencephalus.

\section{METHODOLOGY}

We suppose that within a certain time span (e.g., a year) there are $k$ sectors (e.g., 12 months), and that in sector $i$ there are $n_{i}$ events (e.g. $\omega$ congenital malformations) from a population at risk of size $m_{i}$ (e.g., total births); the total number ofo events is $N=\Sigma n_{i}$ and the total population at risk邑 is $M=\Sigma m_{i}$. Following Edwards, we represent ${ }^{+}$ 


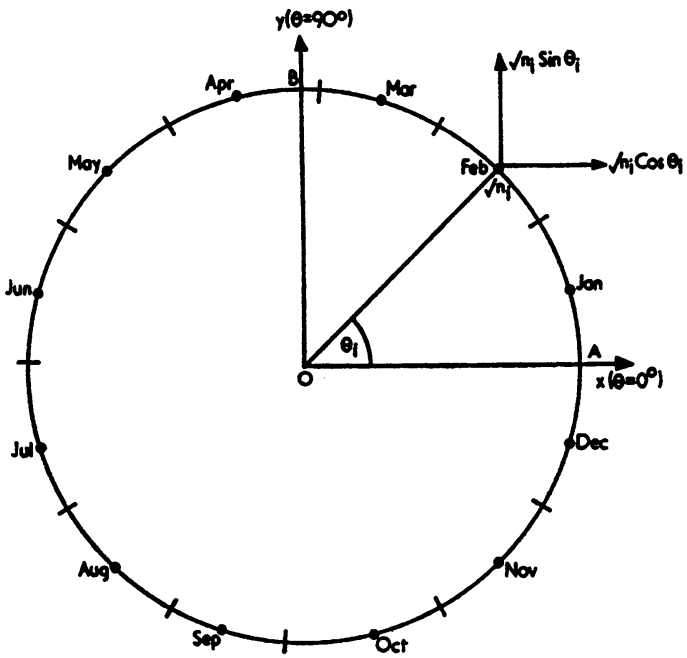

Froure. Typical components of moments about orthogonal axes $O A$ and $O B$.

the data by weights $\sqrt{ } n_{i}$ placed around a unit circle at points corresponding to the sector midpoints at angles $\theta_{i}$ to an arbitrary diameter (e.g., $\mathrm{OA}$, the diameter through 1 January; see Figure). The moment about this diameter of all the weights is $\Sigma \sqrt{ } n_{i}$ Sin $\theta_{i}$, and also considering the moment $i$

about the orthogonal diameter, the sample centre of gravity of the system may be seen to be $(\bar{x}, \bar{y})$ where

$$
\begin{aligned}
& \bar{x}=\sum_{i=1}^{k} \sqrt{ } n_{i} \operatorname{Cos} \theta_{i} / W \\
& \bar{y}=\sum_{i=1}^{k} \sqrt{ } n_{i} \operatorname{Sin} \theta_{i} / W
\end{aligned}
$$

and where $W=\Sigma \sqrt{ } n_{i}$, the total weight. On the null hypothesis, the expected number of events in a sector is proportional to the population at risk in that sector, i.e., $E\left(n_{i}\right)=N m_{i} / M$. We will suppose that the sample is large enough that to calculate the expected values of $\bar{x}$ and $\bar{y}$, we may replace $n_{i}$ by its expectation everywhere in (1) and obtain

$$
\begin{aligned}
E(\bar{x})=\mu_{\bar{x}} & =\underset{i}{\Sigma} \sqrt{ }\left(N m_{i} / M\right) \operatorname{Cos} \theta_{i} / \sum_{i}^{\Sigma} \sqrt{ }\left(N m_{i} / M\right) \\
& =\underset{i}{\Sigma} \sqrt{ } m_{i} \operatorname{Cos} \theta_{i} / \sum_{i}^{\Sigma} \sqrt{ } m_{i}
\end{aligned}
$$

and similarly

$$
E(\bar{y})=\mu_{\bar{y}}=\underset{i}{\Sigma} \sqrt{ } m_{i} \operatorname{Sin} \theta_{i} / \sum_{i}^{\Sigma} \sqrt{ } m_{i}
$$

If we further suppose that the variance of $\sqrt{ } n_{i}$ is approximately $\frac{1}{4}$, and again replace $n_{i}$ by its expected value in $W$, we obtain from (1) that the variances of the coordinates of the sample centre of gravity are

$$
\operatorname{Var}(\bar{x})=\sigma_{\bar{x}}^{2}=\underset{i}{\Sigma} \notin \operatorname{Cos}^{2} \theta_{i} /\left[\Sigma \sqrt{ }\left(N m_{i} / M\right)\right]^{2}
$$

and

$$
\operatorname{Var}(\bar{y})=\sigma_{\bar{y}}^{2}=\sum_{i} \downarrow \operatorname{Sin}^{2} \theta_{i} /\left[\Sigma \sqrt{ }\left(N m_{i} / M\right)\right]^{2}
$$

On the assumption that $\bar{x}$ and $\bar{y}$ are normally distributed, we may take as a test statistic the quantity

$$
\left(\frac{\bar{x}-\mu_{\bar{x}}}{\sigma_{\bar{x}}^{-}}\right)^{2}+\left(\frac{\bar{y}-\mu_{\bar{y}}}{\sigma_{\bar{y}}^{\bar{y}}}\right)^{2}
$$

which on the null hypothesis is distributed as $\chi^{2}$ with 2 d.f. The distance $d$ of the sample centre of gravity from its null expectation is given by

$$
d^{2}=\left(\bar{x}-\mu_{\bar{x}}\right)^{2}+\left(\bar{y}-\mu_{\bar{y}}\right)^{2}
$$

If it is required to fit a simple harmonic trend to the data, we may suppose that the expected frequency in sector $i$ is proportional to

$$
c_{i}=m_{i}\left[1+\alpha \operatorname{Cos}\left(\theta_{i}-\theta^{*}\right)\right] ;
$$

$\alpha$ is a measure of the amplitude of the cyclic variation, and $\theta^{*}$ gives the direction of the maximum rate, which may be estimated as

$$
\theta^{*}=\operatorname{Tan}^{-1}\left(\frac{\bar{y}-\mu_{\bar{y}}}{\bar{x}-\mu_{\bar{x}}}\right)
$$

If we place equal weights of $w / k$ (where $w$ is a constant) at angles $\theta_{i}$ and radii

$$
r_{i}=\sqrt{ }\left\{m_{i} k\left[1+\alpha \operatorname{Cos}\left(\theta_{i}-\theta^{*}\right)\right] / M\right\}
$$

we may derive

$$
w d=\sum_{i} r_{i} \operatorname{Cos}\left(\theta_{i}-\theta^{*}\right) w / k
$$

If the $r_{i}$ 's are expanded as far as terms in $\alpha$ (which is presumed small), and equation (5) is rearranged, we may estimate $\alpha$ as

$$
\alpha=\frac{2\left[d \sqrt{ }(k M)-\Sigma \sqrt{ } m_{i} \operatorname{Cos}\left(\theta_{i}-\theta^{*}\right)\right]}{\Sigma \sqrt{ } m_{i} \operatorname{Cos}^{2}\left(\theta_{i}-\theta^{*}\right)}
$$


In the case $\theta_{i}=2 \pi i \mid k, m_{i}=M / k, i=1,2, \ldots k$, it may be shown that $\mu_{\bar{x}}=\mu_{\bar{y}}=0$, and that (2), (4) and (6) reduce to the corresponding statistics of Edwards' test, which is a special case of this more general formulation. The argument of Smith (1961) concerning the non-independence of the $n_{i}$ in Edwards' test may be extended to this new method. When the sample size is small, the test of Hewitt et al. may be a preferable alternative, using ranked rates rather than numbers of observations to take account of population size seasonality, subject to the requirement that at least six months have a non-zero number of observations. However, if the populations at risk vary substantially, the implied heteroscedasticity of the associated rates may affect the null distribution of the statistic used in Hewitt's test; further simulation to establish the null distribution would be required for specific problems of this type.

The adequacy of the description of the data afforded by a simple harmonic curve may be evaluated by a goodness-of-fit test using a further $\chi^{2}$ statistic. If expected frequencies $n_{i}$ ' are calculated by

$$
n_{i}^{\prime}=N c_{i} / \Sigma c_{i}
$$

then under the usual assumptions, the statistic

$$
\Sigma\left(n_{i}-n_{i}\right)^{2} / n_{i}{ }^{\prime}
$$

has a $\chi^{2}$ distribution with $(k-1)$ d.f. Wehrung and Hay (1970) describe a hypothetical example where the event frequency is constant over all months except one, where a threefold increase is observed, and Edwards' test shows a significant departure from the null hypothesis; a $\chi^{2}$ test using ( $\Varangle$ shows that these data are quite inappropriatel described by a simple harmonic curve, and therefore do not feel that counter-examples of this. kind constitute a substantial objection to the us of this test.

\section{NUMERICAL EXAMPLE}

In Table $I$ are given monthly frequencies of cases of anencephalus and total births for Canade in the period 1954-62 (Elwood, 1975). A cursory inspection of the data reveals a distinct general excess of total births during the summer months whereas the anencephalus cases demonstrate ne? consistent seasonal pattern.

The various statistical tests discussed in the previous section were applied to these data, and the results are given in Table II. Edwards' method produces a test statistic of 0.80 , which is certainly nob significant assuming a $\chi^{2}$ distribution on 2 d.f.; the estimated maximum incidence rate occurs at $\theta^{*}=-18 \cdot 8^{\circ}$, i.e., in mid-December. Resul from the test proposed above are given as tes $\mathrm{B}$ and $\mathrm{C}$ of Table II, the former assuming motits to be of equal length, and the latter using exacs month lengths; the parameters $d, \theta^{*}$ and $\alpha$ of hithe simple harmonic curve were estimated from equations (3), (4), and (6) respectively. The use exact $\theta_{i}$ values made very little difference to th conclusions as compared with test $B$ with equaly spaced $\theta_{i}$ values; the $\chi^{2}$ statistic of test $B$ w $12 \cdot 48$, indicating a deviation from the null hypothesis of constant rates significant at the $0.5 \%$ level, with an estimated maximum occurring at $\theta^{*}=-7 \cdot 4^{\circ}$, i.e., in late December. For simplicit in test $\mathrm{C}$ the length of February was taken as

TABLE I

\begin{tabular}{|c|c|c|c|c|c|c|c|}
\hline \multirow{2}{*}{\multicolumn{3}{|c|}{ Month }} & \multirow{2}{*}{$\underset{\left(m_{i}\right)}{\text { Total Births }}$} & \multirow{2}{*}{$\begin{array}{c}\text { Anencephalus } \\
\text { Cases }\left(\boldsymbol{n}_{\boldsymbol{i}}\right)\end{array}$} & \multicolumn{2}{|c|}{ Expected Frequencies } & \multirow{2}{*}{$\begin{array}{c}\text { Monthly Rankin } \\
\text { of Rates }\end{array}$} \\
\hline & & & & & (i) & (ii) & \\
\hline $\begin{array}{l}\text { January } \\
\text { February } \\
\text { March } \\
\text { April } \\
\text { May } \\
\text { June } \\
\text { July } \\
\text { August } \\
\text { September } \\
\text { October } \\
\text { November } \\
\text { December } \\
\text { Total }\end{array}$ & $\begin{array}{l}\cdots \\
\cdots \\
\cdots \\
\cdots \\
\cdots \\
\cdots \\
\cdots \\
\cdots \\
\cdots\end{array}$ & $\begin{array}{l}\ldots \\
\cdots \\
\cdots \\
\cdots \\
\cdots \\
\cdots \\
\cdots \\
\cdots \\
\cdots\end{array}$ & $\begin{array}{r}340,797 \\
318,319 \\
363,626 \\
359,689 \\
373,878 \\
361,290 \\
368,867 \\
358,531 \\
363,551 \\
352,173 \\
331,964 \\
336,894 \\
4,229,579\end{array}$ & $\begin{array}{r}468 \\
399 \\
471 \\
437 \\
376 \\
410 \\
399 \\
472 \\
418 \\
448 \\
409 \\
397 \\
5,104\end{array}$ & $\begin{array}{r}430 \cdot 9 \\
427 \cdot 9 \\
423 \cdot 9 \\
420 \cdot 3 \\
418 \cdot 0 \\
417 \cdot 5 \\
419 \cdot 1 \\
422 \cdot 2 \\
426 \cdot 1 \\
429 \cdot 8 \\
432 \cdot 1 \\
432 \cdot 5 \\
5,103 \cdot 9\end{array}$ & $\begin{array}{r}434 \cdot 6 \\
398 \cdot 7 \\
443 \cdot 2 \\
424 \cdot 9 \\
430 \cdot 4 \\
410 \cdot 6 \\
420 \cdot 9 \\
417 \cdot 4 \\
436 \cdot 0 \\
435 \cdot 5 \\
420 \cdot 4 \\
431 \cdot 4 \\
5,104 \cdot 0\end{array}$ & $\begin{array}{r}12 \\
8 \\
10 \\
6 \\
1 \\
3 \\
2 \\
11 \\
4 \\
9 \\
7 \\
5\end{array}$ \\
\hline
\end{tabular}

ANENCEPHALUS AND TOTAL BIRTH FREQUENCIES BY MONTH, CANADA, 1954-62

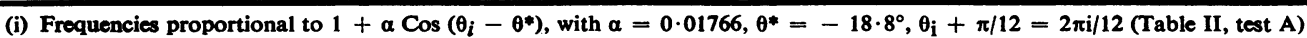
$x^{2}$ Goodness-of-fit statistic $(7)=27 \cdot 24(P<0.005)$

(ii) Frequencies proportional to $m_{i}\left[1+\alpha \operatorname{Cos}\left(\theta_{i}-\theta^{*}\right)\right]$, with $\alpha=0.06014, \theta^{*}=-8 \cdot 1^{\circ}, \theta_{i}$ 's exact (Table II, test $\left.\mathrm{C}\right)$ $\chi^{2}$ Goodness-of-fit statistic $(7)=23.97(P<0.05)$ 
TABLE II

RESULTS OF VARIOUS SIGNIFICANCES TESTS FOR SIMPLE HARMONIC TREND IN ANENCEPHALUS INCIDENCE

\begin{tabular}{|c|c|c|c|c|c|c|}
\hline \multirow{2}{*}{\multicolumn{2}{|c|}{ Test }} & \multicolumn{3}{|c|}{ Estimated Parameters } & \multirow{2}{*}{$\begin{array}{c}\text { Value of } \\
\text { Test Statistic }\end{array}$} & \multirow[b]{2}{*}{ Significance } \\
\hline & & $d$ & $\alpha$ & $\theta^{*}$ & & \\
\hline $\mathbf{A}$ & $\begin{array}{llr}\text { Edwards } \\
\text { frequencies }\end{array}$ & 0.004415 & 0.01766 & $-18 \cdot 8^{\circ}$ & $0 \cdot 80^{1}$ & $P<0.7$ \\
\hline $\mathbf{B}$ & $\begin{array}{l}\left.\text { Walter and Elwood }{ }_{\text {with } \theta_{i}}+2 \pi / 24=2 \pi i 5\right) \\
2 \pi / 12\end{array}$ & 0.01748 & $0 \cdot 07048$ & $-7 \cdot 4^{\circ}$ & $12 \cdot 48^{2}$ & $P<0.005$ \\
\hline C & $\begin{array}{lrr}\text { Walter and } & \text { Elwood } & (1975), \\
\text { with exact } \theta_{\mathbf{i}} & \ldots & \ldots\end{array}$ & 0.01746 & 0.06014 & $-8 \cdot 1^{\circ}$ & $12 \cdot 472$ & $P<0.005$ \\
\hline D & $\begin{array}{l}\text { Edwards (1961), using adjusted } \\
\text { frequencies }\end{array}$ & 0.01733 & 0.06933 & $-6 \cdot 9^{\circ}$ & $12 \cdot 271$ & $P<0.005$ \\
\hline $\mathbf{E}$ & Hewitt et al.(1971) . . & - & - & - & $51^{3}$ & $\mathbf{P}<0.3$ \\
\hline
\end{tabular}

1 Statistic $=\frac{1}{2} \alpha^{2} \mathbf{N}$

2 Statistic given by equation (2)

having null distribution of $\chi^{2}$ with 2 d.f.

3 Largest rank sum of rates for any six-month segment of year; value 51 corresponds to period October-March.

$28 \frac{1}{4}$ days and a total year length of $365 \frac{1}{4}$ days. To be even more correct we should have taken February to have a length of $28+2 / 9$ days with a similar adjustment to the year length to take account of the exact number of leap years occurring in the time period under study; in practice, this refinement makes so little difference to the conclusions that it was decided not to incorporate this additional complexity into the general computer program used for these analyses.

Expected frequencies were calculated for the best simple harmonic curves given by tests $\mathrm{A}$ and $\mathrm{C}$ (see Table I), and these were then compared with the observed frequencies using the $\chi^{2}$ goodness-of-fit test given by equation (7). Although both $\chi^{2}$ statistics indicate significant departures from the fitted curves, that for Edwards' curve is somewhat larger, showing a rather poorer description of the data than that given by the test proposed here. A decomposition of the statistic corresponding to test $\mathrm{C}$ shows that over half the total of 23.97 comes from a large deficit of anencephalics in May and a large excess in August, but that otherwise the observed and expected frequencies are reasonably close.

Several previous authors have employed correction factors when using Edwards' test. One such possibility in the present example would be to replace the $n_{i}$ by $n_{i}^{\prime}=c n_{i} M / k m_{i}$, where $c$ is a scale factor such that $\Sigma n_{i}^{\prime}=N$; the results of such a test are given as test $D$ in Table II. Although the results are very similar to those of tests B and C, we feel that the latter are preferable as they have a less heuristic or empirical basis; it is quite possible that with smaller samples, the differences between the results of tests $C$ and $D$ would be somewhat greater than in the present example.
The non-parametric test of Hewitt et al. (1971) applied to these data gives a maximum rank su $\mathrm{m}$ for the monthly rates as 51 for the six-month period October to March; this value is not significant (test E, Table II). With a substantial amount of data, such as is available here, this test will probably have low power to detect a seasonal trend, unless it is fairly marked (i.e., has a large amplitude) and consistent. Further, this test does not allow one to estimate parameters of the simple harmonic curve even if a significant departure from the null hypothesis is indicated. On the other hand, the argument concerning the differences between the distributions of $\chi^{2}$ and the Edwards' test statistic may also apply to the test proposed here; thus when the sample size is small (say $\mathrm{N}<50$ ), caution should be exercised in the interpretation of the test results, and a nonparametric approach may be a preferable alternative.

\section{REFERENCES}

EDWARDS, J. H. (1961). The recognition and estimation of cyclic trends. Ann. hum. Genet., 25, 83.

Elwood, J. M. (1975). Seasonal variation in anencephalus in Canada. Brit. J. prev. soc. Med., 29, 22.

Hewitt, D., Milner, J., Csima, A., and Pakula, A. (1971). On Edwards' criterion of seasonality and a non-parametric alternative. Brit. J. prev. soc. Med., 25, 174.

SMITH, C. A. B. (1961). Note on the error variance. Ann. hum. Genet., 25, 86.

WeHRUNG, D. A. and HAY, S. (1970). A study of seasonal incidence of congenital malformations in the United States. Brit. J. prev. soc. Med., 24, 24.

Requests for reprints to: Dr. S. D. Walter, Department of Epidemiology and Community Medicine, University of Ottawa, Ontario, Canada. 